\title{
Relación de las calificaciones en la prueba ECOE de la administración catalana con el expediente académico y los resultados en la prueba MIR en estudiantes de Medicina de la UPF-UAB.
}

\section{Relationship of the scores in the OSCE test of the Catalan administration with the academic record and the results in the MIR exam in UPF- UAB Medical students.}

\author{
Juan Gutiérrez ${ }^{1}$, Eva Baillés ${ }^{2}$, Meritxell Girvent ${ }^{3}$, Elisabeth Moyano ${ }^{3}$, Pilar \\ Larramona $^{3}$ y Jorge Pérez ${ }^{3, *}$ \\ ${ }^{1}$ Facultad de Medicina. Universidad Autónoma de Barcelona; ${ }^{2}$ Hospital Nostra Senyora de \\ Meritxell. Andorra \\ ${ }^{3}$ Facultad de Ciencias de la Salud y de la Vida. Universidad Pompeu Fabra \\ 1 Juan Gutiérrez; gutierrez@parcdesalutmar.cat, \\ 2 Eva Baillés; Ebailles@saas.ad, ORCID ID: https://orcid.org/0000-0002-0561-6328 \\ 3 Meritxell Girvent; meritxell.girvent@upf.edu, ORCID ID: https://orcid.org/0000-0001-8244- \\ $\underline{3953}$ \\ 3 Elisabeth Moyano; elisabeth.moyano@upf.edu, ORCID ID: https://orcid.org/0000-0002-0820- \\ $\underline{7220}$ \\ 3 Pilar Larramona; pilar.larramona@upf.edu, ORCID ID: https://orcid.org/0000-0003-2213- \\ $\underline{0140}$ \\ 3 Jorge Pérez; jordi.perez@upf.edu, ORCID ID: https://orcid.org/0000-0003-4433-4734 \\ * Correspondencia: jordi.perez@upf.edu
}

Recibido: 14 de abril de 2020; Aceptado: 26 de mayo de 2020; Publicado: 28 de mayo de 2020

Resumen: En los estudios de Medicina del Campus del Mar de Barcelona (UPFUAB), aunque todavía no se ha implementado la prueba ECOE recomendada por la Conferencia Nacional de Decanos de Facultades de Medicina (CNDFM), todos los estudiantes realizan durante su último año una ECOE diseñada por la administración sanitaria catalana con características similares a la de la CNDFM. Nos planteamos realizar un estudio para establecer la posible concordancia entre los resultados en la prueba ECOE externa y los obtenidos en nuestro centro. También se pretendió establecer la relación entre los resultados en la ECOE y en la prueba MIR. El estudio se realizó con todos los graduados de las cuatro primeras promociones del grado en Medicina de la UPF-UAB que habían realizado la prueba ECOE y la prueba MIR. No se encontraron diferencias entre sexos en los resultados de la ECOE. Tanto en hombres como en mujeres, existió una relación positiva y significativa entre los resultados de la ECOE y los del expediente final y los de la prueba MIR. Los resultados son valorados positivamente ya que el expediente final está en consonancia con el rendimiento en una prueba donde presuntamente se evalúan de forma adecuada las competencias más relevantes que tendrían que tener los graduados en Medicina. La relación positiva entre ECOE y MIR permite cierta tranquilidad hasta que en algún momento pueda implementarse una prueba no exclusivamente cognitiva para el acceso a la formación especializada.

Palabras clave: estudiantes de Medicina; prueba ECOE; prueba MIR; rendimiento académico. 
Abstract: In the Medicine studies of the Campus Mar in Barcelona (UPF-UAB) the OSCE test recommended by the Conference of Deans of Spain (CNDFM) has not yet been implemented, but during their final year, all the students take an OSCE designed by the Catalan health administration with similar characteristics to that of the CNDFM. We plan to carry out a study to establish the possible relationship between the results in the external ECOE test and those obtained in our Medical School. We also tried to establish the relationship between the results in the ECOE and in the MIR test. The study was carried out with all the graduates of the first four promotions of the UPF-UAB degree in Medicine who had taken the ECOE test and the MIR test. No differences between sexes were found in the OSCE achievement. In both men and women, there was a positive and significant relationship between the results of the OSCE and those of the final score and those of the MIR test. The results are positively valued since the final score is in line with the performance in a test where presumably the most relevant competencies that graduates in Medicine should have are adequately evaluated. The positive relationship between OSCE and MIR allows some peace of mind until at some point an exam can be implemented that assesses clinical and communication skills for access to specialized training.

Keywords: academic achievement; ECOE test; medical students; MIR exam

\section{Introducción}

Una de las premisas del Espacio Europeo de Educación Superior (EEES) ha sido la relevancia concedida a la evaluación de las competencias de los estudiantes. Dicho reto ha sido asumido por la Conferencia Nacional de Decanos de Facultades de Medicina (CNDFM) que durante el curso 2011-2012 decidió que, de forma progresiva, todas las facultades introdujeran una prueba durante el último curso de la carrera para evaluar habilidades clínicas y comunicativas. Esta evaluación se haría mediante una prueba tipo Examen Clínico Objetivo y Estructurado (ECOE) de 20 estaciones (1). Dicha prueba, desarrollada por Harden en los años 70 (2) se ha visto adecuada para evaluar el tercer nivel de la pirámide de Miller (3).

A partir de dicha recomendación numerosos centros ya la han implementado (48). La prueba ECOE que se realiza actualmente en las facultades españolas, en su gran mayoría, cumplen los requisitos acordados por la CNDFME recogidos en su página web (http://www.cndmedicina.com/ecoe-2/). Dicha prueba es recibida con gran satisfacción por parte de los alumnos $(5,7)$. También, en Cataluña se viene realizando una ECOE desde 1998 para los graduados en Medicina de las universidades de dicha comunidad $(10,11)$. Las características de dicha prueba son muy similares a las de la recomendada por la CNDFM, ya que consta de 20 estaciones con las mismas 8 áreas competenciales: Anamnesis, Exploración clínica, Habilidades técnicas, Habilidades de comunicación, Juicio clínico, Prevención, Relaciones interprofesionales y Aspectos éticos (11).

Al finalizar sus estudios, la mayoría de los graduados en Medicina de nuestro país opta a ingresar a la formación especializada a través de la prueba de acceso al programa de Médicos Internos Residentes (MIR). Este examen ha sido reconocido por sus ventajas, especialmente por la objetividad que garantiza la equidad $(12,13)$, pero también ha recibido críticas. Principalmente ha sido cuestionado por su orientación casi exclusivamente cognoscitiva, obviando a la evaluación de ciertas competencias clínicas y de comunicación (12-15).

En los estudios de Medicina del Campus del Mar de Barcelona, compartidos por la Universidad Pompeu Fabra (UPF) y la Universidad Autónoma de Barcelona (UAB), todavía no se ha implementado la prueba ECOE pero todos los estudiantes realizan 
durante su último año la prueba diseñada por la administración sanitaria catalana. A partir de este hecho nos planteamos realizar un estudio para establecer la posible concordancia entre los resultados en la prueba ECOE externa y los obtenidos en nuestro centro.

Así, el objetivo principal del presente estudio consistió en establecer la relación entre los resultados obtenidos en la prueba ECOE de la administración catalana y los de la formación en los estudios a través del expediente final.

Como objetivo secundario nos propusimos establecer la relación entre la prueba ECOE que evalúa competencias clínicas y de comunicación (tercer nivel de la pirámide de Miller) y la prueba MIR centrada fundamentalmente en aspectos cognoscitivos (primer y segundo nivel de la pirámide).

\section{Métodos}

\subsection{Participantes}

El estudio se realizó con todos los graduados de las cuatro primeras promociones del grado en Medicina de la UPF-UAB que habían realizado la prueba ECOE y la prueba MIR. Los participantes se graduaron en los cursos 2013-14 ( $N=41), 2015-15(\mathrm{~N}=39)$, 2016-17 ( $\mathrm{N=49)}$ y 2017-18 ( $\mathrm{N}=59)$. De los 188 participantes 53 fueron hombres y 135 mujeres. No todos los graduados participaron en la prueba MIR, en cuyo caso fueron 47 hombres y 124 mujeres.

\subsection{Material}

Para la realización del estudio se registraron, para todos los participantes, las calificaciones obtenidas en la prueba ECOE realizada y en el expediente académico en su versión cuantitativa (de 0 a 10), así como el número de orden obtenido en la prueba MIR.

\subsection{Procedimiento}

Después del registro comentado, se procedió a ordenar a los participantes, de mejor a peor resultado, a partir de las notas obtenidas en la ECOE y en el expediente, y en el número de orden obtenido en el MIR. Así, para el presente estudio se generaron cinco variables: Notas obtenidas en la prueba ECOE, notas en el expediente decimal (EXP), calificación ordinal en la prueba ECOE (ECOE-O), calificación ordinal en el expediente académico (EXP-O) y calificación ordinal en la prueba MIR (MIR-O). Las calificaciones ordinales fueron realizadas dentro de cada promoción y también para toda la muestra.

Seguidamente se procedió a determinar las puntuaciones medias y desviaciones típicas en la variable ECOE, realizándose comparaciones entre graduados y graduadas. Posteriormente se procedió a establecer correlaciones entre las variables de estudio, de Pearson entre las variables ECOE y EXPE, y de Spearman para las variables ordinales ECOE-O, EXP-O y MIR-O.

Finalmente, para la muestra total se establecieron tres grupos a partir del resultado en la prueba MIR. El tercio superior tenía los mejores resultados, con números de orden inferiores a 1400 (Superior), el tercio intermedio, con números entre 1400 y 3400 (Medio) y el tercio inferior con los números más elevados, superiores a 3400 (Inferior). Establecidos los grupos se procedió a la comparación de medias de las variables ECOE y EXP mediante la prueba ANOVA con la prueba de contraste de Bonferroni. 


\section{Resultados}

En los resultados de la prueba ECOE no se encontraron diferencias entre sexos, los varones $(6,46 ; \mathrm{DT}=0,8)$ y las mujeres $(6,43 ; \mathrm{DT}=0,7)$ tuvieron actuaciones semejantes.

Tabla1. Coeficientes de correlación entre los resultados en la ECOE respecto a los del Expediente Académico y a la prueba MIR

\begin{tabular}{|c|c|c|c|}
\hline & $\begin{array}{c}\text { ECOE - EXP } \\
\text { Pearson }\end{array}$ & $\begin{array}{c}\text { ECOE-O-EXP-O } \\
\text { Spearman }\end{array}$ & $\begin{array}{l}\text { ECOE-O-MIR-O } \\
\text { Spearman }\end{array}$ \\
\hline 1a Promoción & $-0,19$ & $-0,17$ & $-0,17$ \\
\hline 2a Promoción & $0,33 * * *$ & $0,46 * * *$ & $0,51 * * *$ \\
\hline 3a Promoción & $0,80 * * *$ & 0,78 *** & 0,51 *** \\
\hline 4ª Promoción & $0,75 * * *$ & $0,75 * * *$ & $0,70 * * *$ \\
\hline Total & $0,40 * * *$ & $0,43 * * *$ & $0,40 * * *$ \\
\hline Hombres & $0,33^{*}$ & 0,39 ** & $0,31 *$ \\
\hline Mujeres & $0,40 * * *$ & $0,45 * * *$ & $0,44 * * *$ \\
\hline
\end{tabular}

$* \mathrm{p}<0,05 ; * * \mathrm{p}<0,01 ; * * * \mathrm{p}<0,001$

En la tabla 1 se muestran los coeficientes de correlación obtenidos entre las variables estudiadas. Como se puede observar, salvo en el caso de la primera promoción, la relación entre ECOE y EXP fue positiva y significativa. A mejor resultado en la ECOE mejor expediente académico. Cuando se realizaron correlaciones no paramétricas, la asociación entre ECOE-O y EXP-O también fue positiva y significativa con la excepción de la primera promoción. El mismo efecto se encontró entre ECOE-O y MIR-O. Con la excepción de la primera promoción, las correlaciones entre ambas variables fueron positivas y significativas. La asociación positiva ECOE con EXP o con MIR fue tanto en hombres como en mujeres.

En la tabla 2 se indican las puntuaciones medias en la prueba ECOE de los grupos realizados a partir de su resultado en la prueba MIR. También se presentan los estadísticos de la prueba ANOVA: los valores de $\mathrm{F}$, los grados de libertad y la significación estadística. Entre corchetes se presentan las diferencias entre grupos de la prueba de contraste Bonferroni.

Tabla 2. Puntuaciones medias y desviaciones típicas en ECOE de los grupos generados a partir del resultado en la prueba MIR. Se presentan los resultados de la prueba ANOVA

\begin{tabular}{|c|c|c|c|}
\hline $\begin{array}{c}\text { Resultado en } \\
\text { MIR }\end{array}$ & Total & Hombres & Mujeres \\
\hline 1. Superior & $6,80(0,6) \mathrm{n}=56$ & $6,82(0,7) \mathrm{n}=16$ & $6,79(0,6) \mathrm{n}=40$ \\
\hline 2. Medio & $6,48(0,6) \mathrm{n}=57$ & $6,40(0,6) \mathrm{n}=21$ & $6,52(0,6) \mathrm{n}=36$ \\
\hline 3. Inferior & $6,27(0,7) \mathrm{n}=58$ & $6,60(0,9) \mathrm{n}=10$ & $6,20(0,6) \mathrm{n}=48$ \\
\hline Total & $6,51(0,7) \mathrm{n}=171$ & $6,59(0,7) \mathrm{n}=47$ & $6,49(0,6) \mathrm{n}=124$ \\
\hline
\end{tabular}

Total: $\mathrm{F}=9,95(\mathrm{gl}=2,168) \mathrm{p}<0,001[1>2,3]$; Hombres: $\mathrm{F}=1,72(\mathrm{gl}=2,44) \mathrm{NS}$;

Mujeres: $\mathrm{F}=10,30(\mathrm{gl}=2,121) \mathrm{p}<0,001[1>3]$

Aparecieron puntuaciones superiores en función del mejor resultado en el examen MIR. Los graduados que tuvieron mejor resultado en la prueba MIR también los obtuvieron en la prueba ECOE, siendo significativos para la muestra total y para la muestra de mujeres. Con toda la muestra, el grupo superior puntuó significativamente mejor que los otros dos, donde no hubo diferencias significativas entre ellos. En la muestra de mujeres, las egresadas del grupo superior tuvieron puntuaciones significativamente superiores al grupo inferior. 
En la tabla 3 se muestran las notas medias del expediente académico de los grupos realizados a partir de su resultado en la prueba MIR. También se presentan los resultados de la prueba ANOVA en los mismos términos de lo comentado en la tabla anterior.

Tabla 3. Puntuaciones medias y desviaciones típicas en EXP de los grupos generados a partir del resultado en la prueba MIR. Se presentan los resultados de la prueba ANOVA.

\begin{tabular}{|c|c|c|c|}
\hline $\begin{array}{c}\text { Resultado en } \\
\text { MIR }\end{array}$ & Total & Hombres & Mujeres \\
\hline 1. Superior & $8,41(0,5) \mathrm{n}=56$ & $8,33(0,6) \mathrm{n}=16$ & $8,45(0,5) \mathrm{n}=40$ \\
\hline 2. Medio & $7,92(0,4) \mathrm{n}=57$ & $7,87(0,6) \mathrm{n}=21$ & $7,95(0,4) \mathrm{n}=36$ \\
\hline 3. Inferior & $7,55(0,4) \mathrm{n}=58$ & $7,43(0,4) \mathrm{n}=10$ & $7,58(0,4) \mathrm{n}=48$ \\
\hline Total & $7,96(0,6) \mathrm{n}=171$ & $7,94(0,6) \mathrm{n}=47$ & $7,97(0,6) \mathrm{n}=124$ \\
\hline
\end{tabular}

Total: $\mathrm{F}=57,04(\mathrm{gl}=2,168) \mathrm{p}<0,001[1>2>3]$; Hombres: $\mathrm{F}=13,02(\mathrm{gl}=2,44)$ $\mathrm{p}<0,001$ [1>2>3]; Mujeres: $\mathrm{F}=46,13(\mathrm{gl}=2,121) \mathrm{p}<0,001[1>2>3]$

Los egresados que tuvieron mejor resultado en la prueba MIR fueron los que tenían mejor expediente. Por el contrario, los del grupo de peor resultado fueron los que tenían un expediente inferior. Dicho efecto fue tanto para la muestra de hombres como de mujeres y siempre se encontraron diferencias significativas entre todos los grupos.

\section{Discusión}

Como se señaló anteriormente, la CNDFM decidió que se debería implementar una ECOE en el último curso de los estudios de Medicina y que serían las propias facultades las que, en función de sus posibilidades, las introdujeran. En los estudios de Medicina del Campus del Mar (UPF-UAB) no se había implementado dicha prueba, pero sus estudiantes en el último curso realizan una ECOE realizada por la administración sanitaria catalana. Siendo conscientes de dicha situación, estábamos interesados en conocer la relación que podría existir entre los resultados en la ECOE citada respecto a los del proceso educativo final del centro y con los obtenidos en la prueba de acceso al programa MIR. Hemos de señalar que tanto la prueba ECOE de la administración catalana como el examen MIR durante los cuatro años del estudio tuvieron las mismas características.

Los resultados en la ECOE fueron muy similares entre varones y mujeres, mostrando una vez más unos resultados de aprendizaje prácticamente idénticos. Ello está en consonancia con otros estudios previos donde no existieron diferencias entre sexos en el expediente final de los graduados en Biología Humana (16) o de Medicina (17) que cursan parte de sus estudios de forma conjunta (18).

En referencia a la relación entre ECOE y EXP, los resultados indican una relación positiva. Salvo en el caso de la primera promoción, todas las correlaciones fueron positivas y significativas. No tenemos una explicación clara sobre la no relación en la primera cohorte de graduados pero ésta queda patente en el resto de promociones, especialmente en las dos últimas. También la relación positiva aparece tanto en hombres como en mujeres, de forma más clara en este último colectivo. En un estudio realizado en una Escuela de Medicina Militar de Estados Unidos también se encontró una asociación positiva entre ECOE y Expediente global, si bien más discreta que en nuestro caso (19). 
A pesar de que la relación encontrada en nuestro estudio puede ser considerada de moderada, la información es valorada positivamente ya que los resultados finales de los graduados están en consonancia con los obtenidos en una prueba donde presuntamente se evalúan de forma adecuada las competencias más relevantes que han de tener los graduados en Medicina.

Con respecto a la relación entre ECOE y MIR, los resultados son semejantes a los obtenidos con la variable EXP. No aparece asociación en la primera promoción, pero sí, de forma positiva y significativa, en el resto. Dicha relación positiva, nuevamente, aparece en graduados y, con más fuerza, en egresadas. También se observó que los estudiantes con mejor resultados en la prueba MIR eran los que, anteriormente, habían tenido las mejores puntuaciones en la prueba ECOE, especialmente en mujeres, y mejor expediente académico. Nuestros resultados son coincidentes con otros realizados en una Escuela de Medicina Militar (20) o en la Universidad de Harvard (21) donde se encontraron correlaciones positivas y significativas entre ECOE y los exámenes de acceso a la práctica profesional en Estados Unidos (USML). Así mismo, el hecho de que las personas con mejores resultados en la prueba MIR tuvieran mejores expedientes reafirma la relación positiva entre expediente y resultados en el examen MR encontrada en un estudio anterior (17).

Creemos que estos resultados son de interés para aportar información al debate sobre el tipo de examen MIR. A pesar de su reconocimiento (12-13), la principal crítica al examen actual es su carácter cognitivo, explorando solamente los dos niveles más bajos de la pirámide de Miller (12-14). Es por lo que se aboga por introducir alguna prueba para evaluar niveles más altos, cosa que podría hacer una prueba ECOE (11). La realidad es que en la actualidad dicha prueba no existe y por ello pensamos que los resultados encontrados son de sumo interés. Al menos con los graduados de los estudios de Medicina (UPF-UAB) hemos visto que existe una importante concordancia entre resultados de evaluación de los dos niveles más bajos de la pirámide (MIR) con otros más elevados (ECOE) hecho que permite cierta tranquilidad hasta que en algún momento puedan implementarse pruebas para evaluar también competencias clínicas y de comunicación. Se podría considerar que nuestros resultados podrían ser extrapolables al resto de los estudios de Medicina de las Universidades Españolas. De todos modos, este supuesto necesitaría confirmación con un estudio más general.

Consideramos que sería muy importante introducir pruebas de ECOE en el grado UPF-UAB, incluso en cursos anteriores al último, pero los resultados obtenidos en este estudio permiten cierta tranquilidad para los responsables académicos de nuestros estudios de Medicina mientras se implementen dichas pruebas en sus procesos formativos. A pesar de lo anterior, ante la relación positiva moderada que hemos encontrado entre ECOE y examen MIR, siempre que fuera posible sería oportuno introducir las ECOE en las pruebas de acceso a la formación especializada MIR.

\section{Conclusiones}

Los resultados del estudio con las cuatro promociones de graduados en Medicina (UPF-UA) permiten establecer las siguientes conclusiones:

- El rendimiento en la prueba ECOE de la administración catalana fue similar en hombres y en mujeres.

- Se encontró una relación positiva y significativa entre el rendimiento en la prueba ECOE y el global de los estudios. 
- Existió una relación positiva y significativa entre el rendimiento en la prueba ECOE y el resultado en la prueba MIR.

Financiación: No ha habido financiación

Declaración de conflicto of interés: Los autores declaran no tener ningún conflicto de intereses

\section{Referencias}

1. García-Estañ J. Prueba nacional de evaluación de competencias clínicas de la Conferencia nacional de decanos de facultades de medicina de España. FEM, 2013, 16 (Suppl 3), S59S62. DOI: $10.33588 / \mathrm{fem} .16 \mathrm{~S} 03.697$

https://www.educacionmedica.net/pdf/revista/16S03/R_FEM_16_S03.pdf

2. Harden RM, Stevenson M, Downie WW, Wilson GM. Assessment of clinical competence using objective structured examination. $\mathrm{Br}$ Med J, 1975, 1(5955), 447-451. https://www.bmj.com/content/bmj/1/5955/447.full.pdf (visitado en 31 de marzo de 20120).

3. Miller G. The assessment of clinical skills/competence/performance. Acad Med, 1990, 65 (9), S63-S67. https://journals.lww.com/academicmedicine/toc/1990/09000

4. Botella C, Moreno M, Fernández-Villacañas MA, García-Estañ J. Formularios para el proceso online de evaluaciones clínicas objetivas estructuradas (ECOE). FEM, 2017, 20(4), 199-204.

https://www.educacionmedica.net/pdf/revista/2004/FEM_2004_199_O_2517013_Garcia.pdf

5. Sánchez-Ferrer F, González de Dios J, Juste-Ruiz M, Compañ AF, Ramos JM. Encuesta de percepción de la evaluación clínica objetiva y estructurada (ECOE) por los estudiantes de sexto grado en medicina de la Universidad Miguel Hernández. FEM, 2017, 20 (3), 137-140. DOI:10.33588/fem.203.893.https://www.educacionmedica.net/pdf/revista/2003/ FEM_2003 137 O_2517005_Sanchez.pdf

6. García-Puig J, Vara-Pinedo F, Vargas-Núñez JA. Implantación del Examen Clínico Objetivo y Estructurado en la Facultad de Medicina de la Universidad Autónoma de Madrid. Educ Med, 2018, 19 (3), 178-187. DOI: 10.1016/j.edumed.2017.01.003

https://www.elsevier.es/es-revista-educacion-medica-71-articulo-implantacion-del-examenclinico-objetivo-S1575181317300232

7. Ramos J, Martínez-Mayoral M A, Sánchez-Ferrer F, Morales J, Sempere T, Belinchón I, Compañ AF. Análisis de la prueba de evaluación clínica objetiva estructurada (ECOE) de sexto curso en la Facultad de Medicina de la Universidad Miguel Hernández de Elche. Educ Med, 2019, 20 (Suppl 1), 29-36. DOI: 10.1016/j.edumed.2017.07.020 https://www.elsevier.es/es-revista-educacion-medica-71-articulo-analisis-prueba-evaluacionclinica-objetiva-S1575181317301584

8. Fernández-Villacañas MA, Moreno-Cascales M, Alemán-Belando S, Matías-Sánchez M, Flores-Funes D, Botella C, García-Estañ J. Simultaneous evaluation of communication skills by standardized patients and medical evaluators. Med Univ, 2019, 2(2), 36-38. DOI: 10.2478/medu-2019-0007

https://content.sciendo.com/view/journals/medu/2/2/article-p36.xml

9. Alemán Belando S, Marín Silvente C, Soriano Giménez V, González Gil A, Flores Funes D, Ramírez Romero P, Cascales Campos PA. Cómo perciben los estudiantes del primer curso clínico del Grado en Medicina la prueba de Evaluación Clínica Objetiva Estructurada (ECOE). Educ Med, 2019 (Suppl 2), 144-147. DOI: 10.1016/j.edumed.2018.02.005 https://www.elsevier.es/es-revista-educacion-medica-71-articulo-como-percibenestudiantes-del-primer-S1575181318300925

10. Rubiano EK, Ricarte Díez JI, Juncosa Font S, Martínez Carretero JM. Evaluación de la competencia clínica de las facultades de medicina de Cataluña, 1994-2006: evolución de los formatos de examen hasta la evaluación clínica objetiva y estructurada (ECOE). Med Clin, 
2007, 129 (20), 777-784. DOI: 10.1157/13113768 https://www.elsevier.es/es-revistaeducacion-medica-71-articulo-como-perciben-estudiantes-del-primer-S1575181318300925

11. Roma J. La prova d'avaluiació de competències (ACOE) als estudiants que acaben Medicina a Catalunya: resultats i perspectives de futur. Ponencia presentada en Jornada AQU. Com millorar la formació dels metges. Barcelona, 15 marzo 2017. http://www.aqu.cat/tallers/opcupadors medicina.html\#.XmjIVKhKjIU

12. Vázquez G, Murillo-Cabezas F, Gómez J., Martín C, Chaves J, Peinado JL. El examen MIR, su cambio como una opción estratégica. Educ Med, 2008, 11 (4): 203-206. DOI:10.33588/fem.114.483 https://www.educacionmedica.net/pdf/revista/1104/1104_203_206.pdf

13. Pérez-Jiménez F. La formación MIR como frontera de la licenciatura de medicina. Educ Med, 2009; $12 \quad$ (Supl 3), S35-S37. DOI: 10.33588/fem.12S03.538 https://www.educacionmedica.net/pdf/revista/12S03/12S03 1_53.pdf

14. Lobato RD, Lagares A, Villena V, Alen JF, Jiménez-Roldan L, Munárriz PM et al. El método de selección de los residentes en España. Análisis del examen MIR y propuesta de una nueva metodología. Neurocirugía 2015; 26 (2), 53-63. DOI: 10.1016/j.neucir.2015.02.001 https://www.revistaneurocirugia.com/es-el-metodo-seleccion-residentes-espana--articuloS1130147315000081

15. Lobato RD, Villena V, Álvarez-Sala JL, García Seoane J, Rubio R, Fernández A, GarcíaCasasola G, Lagares A. La enseñanza de la práctica clínica en la Facultad de Medicina de la Universidad Complutense de Madrid. Educ Med, 2017, 18 (Supl 1), 51-56. https://www.elsevier.es/es-revista-educacion-medica-71-resumen-la-ensenanza-practicaclinica-facultad-X1575181317608243

16. Centeno NB, Rodríguez G, Moyano E, Girvent M, Pérez J. Efecto del sexo en el rendimiento académico de los estudiantes de biología biosanitaria de la Universitat Pompeu Fabra. FEM 2019; 22 (6), 269-272. DOI: 10.33588/fem.226.1024 https://www.educacionmedica.net/pdf/ revista/2206/FEM_2206_269_O_2519033_Perez.pdf

17. Baillés E, Girvent M, Moyano E, Pérez J. Relación entre expediente académico y resultado en la prueba MIR en los estudios de Medicina de la UPF-UAB. FEM, 2020; 23 (en prensa).

18. Morante N, Pérez J. Aprendiendo juntos para trabajar juntos. Comparación del rendimiento académico en estudiantes de biología humana y de medicina en la Universitat Pompeu Fabra. FEM, 21(5), 255-258.

https://www.educacionmedica.net/pdf/revista/2105/FEM_2105_255_O_2518030_Perez.pdf

19. Dong T, Saguil A, Artino AR Jr, Gilliland WR, Waechter DM, Lopreaito J, Flanagan A, Durning SJ. Relationship between OSCE scores and other typical medical school performance indicators: a 5-year cohort study. Mil Med, 2012; 177(Supl 9), 44-46. https://academic.oup.com/milmed/article/177/suppl 9/44/4345540

20. Dong T, Swygert KA, Durning SJ, Saguil A, Gilliland WR, Cruess D, DeZee KJ, LaRochelle J, Artino AR Jr. Validity evidence for medical school OSCEs: associations with USMLE $®$ step assessments. Teach Learn Med. 2014; 26(4), 379-386. https://www.tandfonline.com/doi/abs/10.1080/10401334.2014.960294?journalCode=htlm20

21. Simon SR, Volkan K, Hamann C, Duffey C, Fletcher SW. The relationship between secondyear medical students' OSCE scores and USMLE Step 1 scores. Med Teach. 2002; 24 (5), 535-539. ㄴtps://www.tandfonline.com/doi/pdf/10.1080/0142159021000012586? casa token=3nSFuxnbyTIAAAAA:UcQhgEHN5qDDP7dsvsdC5771DwK6 huqwaTfcIB1nc8 OkBNaor481JUB7evrXM0Z17NfZtCUzLl

2020 por los autores. Enviado para su publicación en acceso abierto bajo los términos y condiciones de la licencia Creative Commons Attribution (CC BY) (http://creativecommons.org/licenses/by/4.0/). 